\title{
Pricing access to Internet service providers
}

\author{
Julian Wright * \\ Department of Economics, Faculty of Arts and Social Sciences, National University of Singapore, \\ AS2 Level 6, 1 Arts Link, Singapore 117570, Singapore
}

Available online 3 March 2004

\begin{abstract}
This paper studies the efficient pricing of termination services for calls that are made to Internet service providers (ISPs). A model is presented in which an incumbent phone network is obligated to provide local calls at a regulated per-minute price while entrant carriers compete to terminate ISP-bound calls. This model is used to understand the problems of regulatory arbitrage that arise when termination charges for ISP-bound calls are not regulated or set reciprocally. Different solutions to the pricing of terminating ISP-bound calls are evaluated.
\end{abstract}

(C) 2004 Elsevier B.V. All rights reserved.

JEL classification: L50; L96

Keywords: Access pricing; Internet; Telecommunications; ISPs

\section{Introduction}

Until recently, termination charges for ISP-bound calls in the US were set at the same level as for normal voice traffic. With capped retail prices for local calls, this led to large financial transfers from incumbent local exchange carriers (ILECs) to competing local exchange carriers (CLECs). ${ }^{1}$ Some CLECs signed up ISPs simply to cash in on receiving termination payments from the incumbent carriers. Reciprocity implied

\footnotetext{
${ }^{*}$ Tel.: +65-6874-3949; fax: +65-6775-2646.

E-mail address: jwright@nus.edu.sg (J. Wright).

${ }^{1}$ According to Randolph (2000), ILECs in the US have given entrant carriers billions of dollars in compensation for ISP-bound calls.
} 
CLECs could recover termination charges based on the cost of terminating voicebound traffic even though ISP-bound traffic is typically long-held, uniform in nature, and geographically concentrated, so that the average cost per-minute of terminating ISP-bound traffic is relatively low. With capped retail prices, ILECs could not pass on these inflated termination charges to end-users. Essentially, the competing carriers exploited an arbitrage opportunity arising from inconsistent regulatory obligations.

In the face of this arbitrage problem, many incumbent carriers withheld payments. In 1999, the FCC stepped in, claiming jurisdiction over the settlement of ISP-bound calls by classifying them as interstate in nature. ${ }^{2}$ In 2001 , the FCC announced it was shifting from reciprocal compensation to an interim cost-based approach, in a transitional move towards a more general "bill and keep" approach to intercarrier compensation (FCC, 2001a,b). Intercarrier compensation for ISPbound traffic was capped at a rate of 0.15 cents per-minute for the second half of 2001, 0.1 cents per-minute for the 18 months thereafter, and 0.07 cents per-minute beyond this point (or until further FCC action). ${ }^{3}$ Any additional costs incurred by carriers had to be recovered directly from end-users.

In this paper, we provide a simple model of ISP competition that incorporates the demand for termination services generated from ISP-bound calls. A key feature of the model is that it assumes the ILEC is required (by regulations) to originate local calls at a fixed retail price. In equilibrium, we find the total price consumers face for Internet dialup includes this regulated price of local calls, but it also depends negatively on the termination charge that is set for terminating ISP-bound calls. ${ }^{4}$ Thus, the total price consumers face for Internet dial-up depends on how local calls are regulated and how termination services are regulated on competing exchanges. The model is used to evaluate alternative approaches to regulating the termination charges for ISP-bound calls.

Using our model, we first characterize the efficient pricing of termination services for ISP bound calls, assuming ISP traffic is not required to provide any contribution to the incumbent's fixed costs. We find welfare is maximized when the termination charges of entrant networks are set equal to the incumbent's (regulated) retail price for local calls, less the cost the incumbent incurs in originating such calls. Assuming a competitive ISP market, this ensures Internet users face the true cost of Internet access. This only corresponds to cost-based termination charges in the unlikely case that the retail price the incumbent charges for local calls is already set at the marginal cost of ISP-bound calls. When the ILEC is required to offer free local calling, this implies CLECs should in fact compensate ILECs for the cost of originating ISP-bound calls.

\footnotetext{
${ }^{2}$ The FCC has jurisdiction over calls between states, while each state has jurisdiction for calls within its borders. Provided ISP-bound calls are viewed as calls that are interstate in nature, the FCC can regulate these rather than relying on the decisions of state commissions.

${ }^{3}$ Pricing the termination of ISP-bound calls has been a contentious issue in many other countries, including for example Australia, Finland, Germany, Italy, New Zealand and the UK.

${ }^{4}$ Consistent with this, several months after the ILEC in New Zealand (Telecom New Zealand) renegotiated a deal with its largest competitor (Clear Communications) that allowed it to avoid termination payments for ISP-bound calls, several formerly free ISPs (including Freenet, i4free, and zfree) either scaled back operations or started charging for Internet dial-up.
} 
We explain why a bill and keep solution may be a reasonable alternative if the retail price of local calls is (close to) zero, while selling wholesale access of the incumbent's origination facilities to CLECs may be a reasonable alternative if the retail price of local calls is high (above-cost). We also explain why allowing competing terminating networks to freely set termination charges is likely to result in the worst outcome, even if the ILEC is allowed to set a (non-discriminatory) retail price for local calls.

The paper has implications for the existing literature on two-way interconnection, which typically assumes two symmetric interconnected networks for which reciprocity of termination charges applies (see Armstrong, 1998; Laffont et al., 1998). With the growing importance of ISP-bound calls, the arbitrage problem detailed in this paper undermines the reciprocity principle when applied uniformly to all types of calls, and suggests other solutions need to be considered. Carter and Wright (2003) suggest ISP-bound calls be treated separately (as the FCC now does), given they are not calls between two different parties, and they are one-directional in nature. Treating such calls separately from the standard two-way interconnection problem is the approach adopted in this paper.

The rest of the paper proceeds as follows. In Section 2, we study the case of competing ISPs and a regulated local incumbent, exploring the implications of reciprocity and characterizing the efficient solution. Section 3 then considers a variety of different approaches to dealing with ISP termination in light of the model. Section 4 briefly concludes.

\section{A model of ISP dial-up}

An important recent development in the structure of local calls is that an increasing proportion of them are for ISP dial-up purposes. An ISP-bound call requires three distinct services - local origination, termination to the ISP's modem banks, and the ISP service (modem banks, access to the Internet and customer service). When people connect to their ISPs, their calls have the following features. Calls to ISPs are one-way in nature and are typically much longer than normal voice calls. Calls typically originate on the incumbent's network. Not only does this occur because most households subscribe to the ILEC's network, but more importantly because typically it is only the regulated incumbent that is obligated to carry these potentially unprofitable calls. The proportion of ISP-bound calls that terminate on each carrier's network depends on how many ISPs each of the carriers has signed up, and how many customers each of these ISPs serves. ISP-bound calls are also likely to be cheaper to terminate than regular calls. ISP-bound traffic requires less equipment and is more concentrated than other traffic. When people dial up their ISP to connect to the Internet, they also tend to stay logged on for long periods, leading to lower average per-minute costs.

To model this situation, we suppose that all calls terminate on one of two CLECs. ${ }^{5}$ Each of these entrant networks also offers an ISP service directly to

\footnotetext{
${ }^{5}$ Our model assumes the ILEC does not offer its own ISP service. Relaxing this assumption does not affect the main results of this paper, as will be discussed.
} 
customers. As long as the market for ISP dial-up is competitive, our model would also hold in the case CLECs did not own the ISPs. CLECs would still face the same final demand from end-users because ISPs would pass through the CLECs' charges to end-users. However, modeling separate CLECs and ISPs involves additional notation. Thus, for brevity, we assume the CLEC and the ISP are one in the same, and refer to CLECs and ISPs interchangeably. Our model of CLEC competition follows a standard Hotelling framework with two-part tariffs.

We assume the following cost structure for calls. The per-minute cost the incumbent faces for originating or terminating a typical (voice) local call is denoted $c^{\mathrm{V}}$. Each competing carrier incurs a cost $c^{\mathrm{T}}$ per minute for terminating ISP-bound calls on its network (where $c^{\mathrm{T}}<c^{\mathrm{V}}$ ), as well as a cost $c^{\mathrm{I}}$ per minute for offering Internet access and other associated Internet services to its customers. In addition, we assume the ISP incurs a cost of $f$ per customer served. These costs include the costs that the ISPs may have to pay to obtain access to the Internet backbone.

The utility derived from subscribing to network $i$ is assumed to be $u\left(q_{i}\right)+\theta_{i}+v_{0}$ where $q_{i}$ is the number of minutes of dial-up to the Internet, $\theta_{i}$ measures the additional benefits of belonging to network $i$, and $v_{0}$ is some additional benefit of the service that does not depend on the network chosen or the length of dial-up. For simplicity, we suppose there is a fixed number $N$ of consumers who want Internet access, where $v_{0}$ is sufficiently large so that their utility is non-negative in equilibrium. We will later discuss what happens when there is only partial participation by households in the Internet access market.

Consumers are endowed with a value of $x$ which is drawn from a uniform distribution on the interval $[0,1]$, while the networks are located at 0 and 1 . A consumer with value $x$ receives extra benefits

$$
\theta_{1}=\frac{1-x}{2 \sigma} \text { and } \theta_{2}=\frac{x}{2 \sigma}
$$

from subscribing to networks 1 and 2 respectively. The parameter $\sigma$ measures the degree of substitutability between the networks. For low values of $\sigma$, a firm can price higher than its rival without losing much market share. As $\sigma \rightarrow \infty$, only price differentials matter and the firm with the lowest prices captures the whole market.

Given that households' marginal willingness to pay is homogeneous, firms can do no better than offer two-part tariffs. Consumers face potentially two usage charges. The incumbent is assumed to be required by USO requirements (or other regulations) to offer calls on demand to households at the regulated price $P$ per-minute. The regulated price $P$ is assumed to be fixed (perhaps because it is set in a price-cap) - the ILEC does not have the option of changing it in response to a change in the termination charge set by CLECs. ${ }^{6}$ While local voice calls cost $2 c^{\mathrm{V}}$ per-minute $\left(c^{\mathrm{V}}\right.$ for origination and $c^{\mathrm{V}}$ for termination), the regulated price $P$ can be above or below this cost. ${ }^{7}$ All calls are as-

\footnotetext{
${ }^{6}$ The case where $P$ is determined by the ILEC is analyzed in Section 3.1.

${ }^{7}$ In the UK, and much of Europe, local retail prices can be as much as several US cents per-minute. Within a number of jurisdictions in the US, local-calls set by ILECs are required to be offered for free $(P=0)$.
} 
sumed to originate on the incumbent's fixed-line network. The $i$ th ISP sets a per-minute price, denoted $p_{i}$, and a lump-sum fee (monthly rental), denoted $r_{i}$.

The net utility (absent of network specific benefits) obtained from subscribing to ISP $i$ is then

$$
w_{i}=v_{i}\left(p_{i}+P\right)+v_{0}-r_{i},
$$

where

$$
v_{i}\left(p_{i}+P\right)=\max _{q}\left\{u(q)-\left(p_{i}+P\right) q\right\} .
$$

A consumer located at $x$ will be indifferent between the two networks if

$$
w_{1}+\frac{1-x}{2 \sigma}=w_{2}+\frac{x}{2 \sigma} \text {. }
$$

Solving for $x$, the market share obtained by network 1 is

$$
s_{1}=\frac{1}{2}+\sigma\left(w_{1}-w_{2}\right)
$$

while $s_{2}=1-s_{1}$.

The per-minute termination charge set by the $i$ th CLEC is denoted $a_{i}$. This access price is assumed to be set prior to firms setting their retail prices. The local incumbent's profit on calls to ISPs is

$$
\pi_{0}=N s_{1}\left(P-c^{\mathrm{V}}-a_{1}\right) q\left(p_{1}+P\right)+N s_{2}\left(P-c^{\mathrm{V}}-a_{2}\right) q\left(p_{2}+P\right)-F_{0} .
$$

The total number of calls to each network depends on the number of subscribers to its ISP service $\left(N s_{i}\right)$, and the minutes called by each of these subscribers $(q)$. The latter depends on the per-minute price for Internet usage $\left(p_{i}\right)$ set by the $i$ th ISP, as well as the incumbent's regulated per-minute charge $P$. The term $F_{0}$ represents the net contribution required by the incumbent to its fixed and common costs arising from offering ISP-bound calls.

The entrant network, ISP $i$, generates a profit of

$$
\pi_{i}=N s_{i}\left(p_{i}+a_{i}-c^{\mathrm{T}}-c^{\mathrm{I}}\right) q\left(p_{i}+P\right)+N s_{i}\left(r_{i}-f_{i}\right)-F_{i} .
$$

The first term captures the margins obtained from Internet usage - it measures the per-minute retail charges for the ISP service $\left(p_{i}\right)$, plus termination charges $\left(a_{i}\right)$, less the per-minute costs $\left(c^{\mathrm{I}}+c^{\mathrm{T}}\right)$ of providing the service, multiplied by the number of minutes of calling. The second term is the per-customer margin arising from ISP customers (rentals less per-customer fixed costs). The last term represents the costs of providing the ISP and termination services that do not depend on either volume of traffic or number of customers.

For given termination charges $a_{1}$ and $a_{2}$, the Nash equilibrium prices and rentals are

$$
\begin{aligned}
& p_{i}=c^{\mathrm{T}}+c^{\mathrm{I}}-a_{i}, \\
& r_{i}=f_{i}+\frac{s_{i}}{\sigma} .
\end{aligned}
$$


The ISPs' retail price for Internet access is decreasing in the termination charge for ISP dial-up calls. With higher values of $a_{i}$, ISPs encourage greater Internet usage by lowering their prices, so that they can increase their termination revenues.

We start by characterizing the efficient level of termination charges. Assuming the regulated ILEC price $P$ is given, the efficient level of termination charges will be such that consumers of ISP dial-up face usage prices equal to cost. This will happen if both networks set per-minute termination charges equal to

$$
a_{i}=P-c^{\mathrm{V}} .
$$

Then consumers of ISP dial-up will face usage prices of

$$
p_{i}+P=c^{\mathrm{T}}+c^{\mathrm{I}}-P+c^{\mathrm{V}}+P=c^{\mathrm{V}}+c^{\mathrm{T}}+c^{\mathrm{I}},
$$

which reflect the true marginal costs of ISP dial-up. Thus, the efficient termination charge should be based on the regulated retail price of a call less the cost that the incumbent incurs for originating the call. ${ }^{8}$ Since this does not depend on the CLECs' costs or prices, the socially optimal termination charge is in fact the same across entrant carriers. This result is summarized in our first proposition.

Proposition 1. The efficient termination charge for ISP-bound calls equals the incumbent's (regulated) retail price for a local call, less the cost the incumbent incurs in originating the call.

Proposition 1 says the efficient termination charging regime will reflect the incumbent's retail pricing regime once the costs the incumbent incurs in originating calls are deducted. The idea is to correct any distortion present in local call prices, so that the net usage price for ISP dial-up equals marginal cost. If local call prices are inflated above cost, then this positive margin should be reimbursed to ISPs, and thus their customers, so that users face the true cost of ISP-bound calls. If local call prices are held below cost, then the ISP should be required to cover the cost shortfall, so that its customers face the true cost of ISP-bound calls.

The usage price for Internet access resulting from this access regime would be the same price set by the ILEC if it also offered a competitive ISP service. It would set a two-part tariff, with usage prices set at marginal cost, which are $c^{\mathrm{V}}+c^{\mathrm{T}}+c^{\mathrm{I}}$. Thus, Proposition 1 would still apply if the incumbent itself offered an ISP service. The socially optimal termination charge is competitively neutral between the incumbent and entrant in the ISP market. Moreover, the ILEC or rival ISPs may still offer unmetered Internet dial-up, at least up to some time limit, if such packages are popular with customers and the costs can be recovered through sufficiently high subscription charges for the average user. However, under the proposal, such decisions will be

\footnotetext{
${ }^{8}$ This differs from the efficient component pricing rule (ECPR). For an incumbent that also provides an ISP service, ECPR implies it would offer the ability to re-sell the ISP service (including dial-up) over its network at an access price equal to the total price it charges (say $P+p$ ) less its cost saving from not having to terminate ISP-bound calls $\left(c^{\mathrm{T}}\right)$, and not having to provide the ISP service $\left(c^{\mathrm{I}}\right)$.
} 
based on the underlying costs and benefits of doing so, rather than any artificial subsidy to ISPs.

The above proposal for regulating termination charges focuses on correcting marginal pricing incentives. Provided the ISP market is competitive, the proposal will not affect the CLECs' equilibrium profits (this is also true if we allow for freeentry, which seems reasonable in this market). ${ }^{9}$ If CLECs also offer origination services, assuming they match the incumbent's price of $P$, they will break even on the origination of ISP-bound calls, but will recover fixed costs through subscription fees, and possibly through residential voice calls (depending on the level of $P$ ). This parallels the situation for the ILEC. Another advantage of the above proposal is that the informational requirements of the regulation are minimal. Because most approaches to the regulation of existing ILECs already involve estimation of the cost of local origination (and termination), regulators can make use of existing estimates of $c^{\mathrm{V}}$.

The efficiency result assumes the objective of policymakers is to achieve costbased usage pricing for Internet access. This would be the case if lump-sum or subscriber charges can be used to fully achieve the regulator's other goals. If ISPbound calls are also required to provide a contribution to the fixed and common costs of the incumbent's network (beyond those that might be recovered through any additional monthly rentals), then the optimal termination charge should be lower than that characterized in (6). If, on the other hand, not all consumers subscribe to one of the ISPs, and policymakers want to promote additional participation on the Internet, then one way to do this is to have the termination charge be set higher than that characterized in (6). We assume that the government uses other instruments to achieve these additional objectives, so that when setting the termination charge for ISP-bound calls it takes a neutral approach, only seeking to achieve efficiency in the resulting usage prices. Alternatively, the solution given in (6) can be viewed as a compromise between the need to prevent arbitrage arising from termination charges that are too high, and the need to promote Internet access as much as possible. ${ }^{10}$

Under the approach taken, the efficient termination charge can differ substantially from the reciprocal approach adopted until recently in the US (and some other countries), in which the termination charge is set at the same (regulated) level that applies to calls terminating on the incumbent's own fixed line network. Assuming the incumbent has its termination charge regulated to cost, this implies $a=c^{\mathrm{V}}$. In jurisdictions where local calls are capped at a price below $c^{\mathrm{V}}$ per-minute (for example, with free local calling), efficient termination pricing implies that the terminating

\footnotetext{
${ }^{9}$ The case where CLECs or ISPs can retain some of the margins from higher termination charges is discussed following Proposition 2.

${ }^{10}$ A subsidy for Internet dial-up may be desirable if there is a positive network externality associated with having a greater number of other users who are able to access the network. However, there may be an offsetting congestion effect resulting from any underpricing of Internet dial-up. The nature of these two effects suggests any subsidy would be better aimed at reducing the subscription charge for Internet dial-up rather than reducing usage charges. Moreover, even if for some reason policymakers prefer below-cost usage pricing for Internet dial-up, it is not clear why such a subsidy should be funded by ILECs.
} 
network should be paying the incumbent for origination services provided, and collecting the price to cover this charge from customers directly.

Reciprocity will exactly coincide with the efficient termination price when local calls are priced exactly at cost - that is, when local retail prices are set equal to the cost of originating and terminating a local call $\left(2 c^{\mathrm{V}}\right)$. Often, local calls have their usage price subsidized, which in the context of our model means $p<2 c^{\mathrm{V}}$. If this is the case, then reciprocity will imply the per-minute component of any termination charge will be set above the efficient level defined above. From (4) we can get the following result:

Proposition 2. With retail prices of local calls set below cost, reciprocity implies Internet access will be priced too low (below cost).

The below-cost pricing of ISP dial-up results from a transfer from the incumbent to consumers. When not all the benefits of greater termination revenues flow back to consumers, part of the transfer will remain with CLECs and/or ISPs instead. In this case, the transfer of money could lead to an over-investment in termination services and ISP services. One (partial) explanation for the financial troubles of CLECs and ISPs in the US during the 2001-2002 period could be that they overinvested in providing Internet access services, which were only profitable based on inflated termination charges that have since been reduced as a result of reciprocity being eliminated.

A compelling reason not to allow termination charges for ISP-bound calls to be set too high is if doing so leads to an unbounded arbitrage problem. Such a problem can threaten the sustainability of the incumbent's origination service. For instance, in jurisdictions with free local calling and reciprocity of termination charges, these charges will be set above the cost of terminating ISP-bound calls, and so according to our model the price of Internet dial-up will be $p_{i}+P=c^{\mathrm{T}}+c^{\mathrm{I}}-a_{i}=c^{\mathrm{T}}+c^{\mathrm{I}}-c^{\mathrm{V}}$. Where the cost of Internet services provided with dial-up $\left(c^{\mathrm{I}}\right)$ is sufficiently low compared to the cost of originating the local call $\left(c^{\mathrm{V}}\right), p_{i}+P$ can become negative and terminating carriers may actually pay people to dial-up. By paying people to dial into the Internet, these entrant networks can increase demand for their termination services almost without bound. ${ }^{11}$ In fact, in some cases, networks may need not even incur the cost of providing an Internet service to provide a termination service. This saves them the per-minute cost of $c^{\mathrm{I}}$ and allows them to share some of the margin $c^{\mathrm{V}}-c^{\mathrm{T}}$ with customers. An almost unbounded arbitrage opportunity is created, in which the demand for making calls to the dial-up number becomes potentially very large. As resources are attracted into providing and marketing such schemes, and in providing the origination and termination services associated with them, efficiency

\footnotetext{
${ }^{11}$ Demand will arise from, among other sources, people dialing in overnight or while at work, the use of multiple lines, and people remaining connected to the Internet, rather than disconnecting and dialing back when they take a break.
} 
decreases. In addition to the misallocation of resources, and the resulting distorted investment decisions, congestion of the local network may also occur.

\section{Alternative regulatory solutions}

In the following sections, we consider alternative regulatory solutions for the pricing of call termination in the context of the simple model of ISP bound-calls presented in the previous section. Section 3.1 considers the case in which there are no constraints on the termination charges that competing local exchange carriers can offer. This turns out to be the worse outcome, unless the incumbent local network is given considerable latitude in its own retail pricing of local calls. Section 3.2 contrasts this with the direct regulation of termination charges based on the cost of termination. This leaves open the risk of arbitrage opportunities given the difficulties regulators face of measuring costs accurately. Finally, two alternative approaches are presented - 'bill and keep' in which termination charges are set at zero for ISP call termination is evaluated in Section 3.3, while allowing terminating carriers to buy wholesale access to origination services and thus retail ISP-bound calls themselves is evaluated in Section 3.4.

\subsection{No regulatory constraints on termination charges}

This section considers what would happen if competing entrant networks were free to set any charge they want for terminating local calls. The higher the CLECs' termination charges, the lower the per-minute Internet dial-up charge they will offer. Since each competing carrier can substitute termination revenue for Internet dial-up revenue, this provides an attractive way for them to gain market share. By increasing their termination charge they can lower their per-minute price for Internet usage, increase market share, and yet still capture the same revenue per-customer through termination revenues.

To see how this works, note firm 1's equilibrium market share is

$$
s_{1}=\frac{1}{2}+\frac{\sigma}{3}\left[v\left(c^{\mathrm{T}}+c^{\mathrm{I}}-a_{1}+P\right)-v\left(c^{\mathrm{T}}+c^{\mathrm{I}}-a_{2}+P\right)+f_{2}-f_{1}\right],
$$

where each firm sets a termination charge $a_{i}$. Equilibrium profits are

$$
\pi_{i}=\frac{N s_{i}^{2}}{\sigma}-F_{i}
$$

Then, since

$$
\frac{\mathrm{d} s_{i}}{\mathrm{~d} a_{i}}=\frac{\sigma}{3} q\left(c^{\mathrm{T}}+c^{\mathrm{I}}-a_{i}+P\right)>0,
$$

each firm will want to set their termination charge as high as possible. By doing so, it increases its market share and thus its profit. There is no end to this escalation problem provided the incumbent is still able and required to offer the origination 
service. The higher is a carrier's termination charge, the lower is the price it will want to charge its customers for Internet access, and thus the greater the demand for Internet usage and so its termination services. With demand for its termination increasing in its termination charge, the carrier will always want to increase its termination charge. However, because in any symmetric outcome both carriers increase their termination charges by the same amount, market shares are unchanged in equilibrium. From (8), neither ISP benefits. Rather, the lower prices offered to consumers perfectly offset the increase in termination revenues. The result is a transfer from the incumbent network to those consumers wanting Internet access. ${ }^{12}$

In practice, this escalation of charges will be limited. At some point, if the termination charges are too high, the transfer from the incumbent to entrants would lead the incumbent to close down. More likely, policymakers would intervene well before this happens (as they have done in various countries). The key results are summarized in the following proposition:

Proposition 3. Each entrant network will try to set termination charges as high as possible. The escalation of termination charges is only bounded by the ability of the incumbent to continue to offer origination services, or by the threat of regulatory intervention.

Relying on competition between terminating carriers to solve the ISP reciprocal compensation problem is only likely to make the problem more acute. Moreover, this result does not depend on the incumbent's price being fixed by regulation. If the incumbent is able to price ISP-bound local calls freely, then the above escalation problem will not necessarily be solved. For instance, if the incumbent is allowed to set its own retail price for local phone calls, then provided it cannot price discriminate with respect to the terminating network, each individual terminating network will still face an incentive to increase termination charges above cost, since it shares any reduction in demand from higher prices with all other competing networks. In this case, it is straightforward to show that

$$
\frac{\mathrm{d} s_{i}}{\mathrm{~d} a_{i}}=\sigma\left[q_{i}+\left(q_{j}-q_{i}\right) \frac{\mathrm{d} P}{\mathrm{~d} a_{i}}\right] .
$$

Starting from any symmetric outcome (so $a_{1}=a_{2}$ and $s_{1}=s_{2}$ implies $p_{1}=p_{2}$ and $\left.q_{1}=q_{2}\right)$, it follows that

$$
\left(\frac{\mathrm{d} \pi_{i}}{\mathrm{~d} a_{i}}\right)_{a_{1}=a_{2}}=N q_{i}>0 .
$$

Regardless of the level of the termination charges, each CLEC will want to increase its termination charge above its rival's level. As any increase in the incumbent's

\footnotetext{
${ }^{12}$ In some different models of imperfect competition, the CLECs or ISPs may be able to keep some portion of the increased termination revenues. Also if the incumbent offers its own ISP services, the result of the inflated termination charges is to cause the incumbent to offer lower (below-cost) prices to its own ISP customers, in order to prevent them switching to the rivals - in which case the result is the same.
} 
(uniform) price of local calling will affect the demand for both firms' ISP dial-up service equally, the firm increasing its termination charge is able to offer lower direct charges of ISP dial-up, and achieve an increase in market share. Thus, the escalation of termination charges arises under competition even if the incumbent carrier is free to set prices for local ISP-bound calls, as long as it cannot set discriminatory prices across the rival networks. ${ }^{13}$

Only if the incumbent sets retail prices for ISP dial-up that reflect the termination price $a_{i}$ of each carrier would this escalation not arise. If the ILEC is free to set (differential) retail prices for ISP-bound calls that reflect each CLEC's termination charge, due to two-part pricing, it will set per-minute prices equal to perceived cost $c^{\mathrm{V}}+a_{i}$. The total price paid by consumers for ISP dial-up will be $p_{i}+P=c^{\mathrm{T}}+c^{\mathrm{I}}-a_{i}+c^{\mathrm{V}}+a_{i}=c^{\mathrm{V}}+c^{\mathrm{T}}+c^{\mathrm{I}}$. In this case, the level of the termination charge $a_{i}$ is irrelevant to the total price paid by consumers for ISP dial-up. Any increase in carrier $i$ 's termination charge will just mean its customers will pay more for dial-up (through the ILEC), and pay equivalently less for the ISP service from carrier $i$. Competition will ensure the total price of ISP dial-up is set at the efficient level.

Allowing the incumbent to price discriminate across competing carriers may, therefore, be another solution to the problem of inflated termination charges. If there is insufficient facilities-based local exchange competition to keep retail prices at reasonable levels, this may require some kind of global price-cap on local calls. Such a policy still leaves open the possibility that the incumbent, if it also operates an ISP service itself, may increase the price of ISP dial-up to rival ISPs and lower the price of ISP dial-up to its own customers, in order to meet any global price-cap, while squeezing out rival ISPs. Alternatively, regulators could allow the resale of origination and termination services over the incumbent's network, which with imputation tests, could be used to discipline the incumbent's retail pricing.

\subsection{Cost-based termination charges}

Perhaps the most obvious regulation of termination services is to set them equal to cost. In terms of our model, this means setting $a=c^{\mathrm{T}}$. Apart from the issues of measuring $c^{\mathrm{T}}$, and ensuring this represents best-practice costs, this will only correspond to the efficient termination charge when the regulated price of local calls is set at the cost of originating and terminating an ISP-bound call, so that customers face these costs as well. This is because when $a=c^{\mathrm{T}}$, (4) implies $p_{i}=c^{\mathrm{I}}$ and the price for ISP-bound calls is $p_{i}+P=c^{\mathrm{I}}+P$. So unless $P=c^{\mathrm{V}}+c^{\mathrm{T}}$, consumers will face a price for Internet dial-up which is different from cost. With un-timed local calls, they will only pay $c^{\mathrm{I}}$ per-minute for ISP dial-up.

\footnotetext{
$\overline{13}$ Wright $(2002$, p. 303) shows this result in a similar setting, as a special case of a more general model of fixed-to-mobile termination. See also Section VII(ii) of Wright (2002), which explains the economic differences between fixed-to-mobile termination and the termination of ISP-bound calls considered here.
} 
This policy rules out the arbitrage problem that arises under reciprocity, since there is no margin between terminating cost and revenue to pay subscribers to dialup. However, this assumes costs have been measured precisely. The possibility of arbitrage implies particular care is needed in measuring the termination costs for ISP dial-up. Although when applied appropriately, cost-based termination charges need not lead to an arbitrage problem, they can still lead to transfers away from the incumbent local network. If the amount of termination charges payable to terminating networks on a call of average length exceeds the regulated retail price collected by the incumbent local network, then each additional ISP-bound call will be a loss-making proposition for such a network (even if there is no cost of originating the call). This is true by definition for jurisdictions with free local calling. While this result may also appear to apply to CLECs offering local call origination, because only ILECs are typically required (through USOs) to provide such money-losing calls, it is only the ILECs which will cover the costs of such calls. Where ISP-bound calls increasingly dominate traffic flows, a more extreme solution may be needed.

\subsection{Bill and keep}

One approach to regulating termination charges that is being considered by the FCC is that of 'bill and keep,' in which no termination charges are payable to carriers that terminate ISP-bound calls (see FCC, 2001a). Further support for the bill and keep approach comes with the release of two working papers by the FCC which provide arguments in favor of a bill and keep approach to interconnection more generally (see Atkinson and Barnekov, 2000; DeGraba, 2000). DeGraba suggests the ISP reciprocal compensation problem as an important reason why a bill and keep approach to interconnection is a superior approach more generally - it avoids any problems of regulatory arbitrage.

Given that the costs of terminating ISP dial-up are very small (recall, the FCC's cost-based rates of 0.07 cents per minute), bill and keep is an approximation to costbased termination charges that has the advantage that it is avoids the need to measure costs. Under bill and keep, $a_{1}=a_{2}=0$, and (4) implies $p_{i}=c^{\mathrm{I}}+c^{\mathrm{T}}$. The total price for ISP-bound calls is $p_{i}+P=c^{\mathrm{I}}+c^{\mathrm{T}}+P$. The outcome is optimal if $P=c^{v}$. With $P<c^{v}$, it is desirable to the extent below-cost pricing of local calls is considered appropriate for ISP-bound calls as well. Where local calls are actually priced above cost, this approach (as with cost-based termination charges) can lead to over pricing of ISP-bound calls. The efficient pricing solution described by (6) guards against such possibilities.

It is sometimes argued that setting termination charges to zero would leave little incentive for networks to offer termination services, at least those of high quality. This is not in fact true. Firms can still recover revenue for such services, directly from end-users. However, whether policymakers will find the resulting increase in price of Internet dial-up acceptable is another matter, especially if the usage price of local calls is already non-trivial. In such cases, an alternative approach may be appropriate. 


\subsection{Wholesaling ISP access}

In the UK, as with a number of other European countries, local phone usage charges are allowed to be positive and non-trivial (they can be several pence per minute). The case in which the retail per-minute price for local calls is high (relative to cost) is the most likely situation in which the assumptions underlying our efficiency result in (6) are violated. The likely justification for regulators to allow high perminute retail price for local calls is to provide a contribution to cover the incumbent's fixed and common costs of the fixed-line incumbent while keeping line rental charges low. If this is the case, there is likely to be similar grounds for doing so with ISP traffic. This suggests a termination charge below that implied by (6) may be desired.

In fact, in countries with significant usage prices for local calls, an altogether different approach to the problem of ISP-bound calls has generally been adopted. Typically, the entrant network will buy access to the incumbent's origination service and offer the joint service (local calls and the ISP service), thereby collecting the retail price for the local call and a subscription fee for Internet access. One problem with this approach is that the incumbent could itself offer ISP services on a flat rate basis, but by charging its rivals per-minute charges for origination services, force them to set positive usage charges to cover their costs. Such a scenario has been suggested by the German regulator (Boston, 2000).

One solution to this price-squeeze problem is to have the incumbent provide access on a flat rate basis. British Telecom, under pressure from the British regulatory authorities, introduced wholesale un-metered net access for ISPs and other licensed operators in 2001. ISPs purchase unlimited access 24 hours per day for a simple quarterly port-based fee. A flat rate access charge will allow flat rate Internet access even where there are significant per-minute call charges for local voice calls. However, the economic rationale for flat rate Internet access remains an issue, as flat rate charging raises the possibility of capacity shortages, and other distortions.

\section{Conclusions}

In this paper, we have analyzed the problem of setting termination charges for ISP-bound traffic. We showed that under reciprocity, when the regulated retail price of local calls is set at or below cost, there will generally be below-cost pricing of ISP services. Reciprocity does this by transferring funds from established network providers to competing exchange carriers or ISPs on a usage basis. Since the cost of terminating an ISP-bound call is considerably lower per minute than the cost of terminating a regular local voice call, reciprocity can also lead to arbitrage. Given the problems arising from reciprocity, we explored different regulatory solutions.

We characterized the efficient termination charge to be paid by ILECs to CLECs for terminating ISP-bound calls. This equals the incumbent's retail price for a local call, less the cost the incumbent incurs in originating the call. Provided the market for terminating calls is competitive and ISP-bound calls are not required to contribute to the fixed and common costs of providing other services, this leads to the efficient 
pricing of ISP-bound calls. Given that this solution can imply positive usage charges for Internet access when there are significant underlying usage costs to be funded, this may not be considered desirable by policymakers who may want to protect flat rate Internet access or subsidize Internet usage. With this in mind, we considered other solutions - namely, freely set termination charges, bill and keep, cost based termination charges, and wholesale access to the incumbent's network services.

We found that if entrant carriers are allowed to freely set termination charges, an escalation of termination charges will ensue. Each terminating carrier will seek to set their termination charge above their rivals. We show this is still true even if the incumbent is free to set the price of ISP-bound local calls, provided it sets the same price regardless of the terminating carrier. Thus, allowing termination charges to be set in the absence of regulation will only lead to even higher termination charges compared to those arising under reciprocity regardless of how competitive the ISP market is. Only when the local incumbent can set differential local call prices to discriminate against terminating carriers that charge more, will these carriers be constrained in raising their termination charges.

We found that if retail prices are regulated to offer free local calling (as is often the case in the US), low ISP usage prices can be maintained using a bill and keep approach. Because this solution does not lead to transfers from the incumbent network to entrants, the arbitrage problem symptomatic of reciprocity is resolved. Where local call prices have a positive but small usage component, cost-based termination charges can achieve the objectives of low Internet usage prices without leading to arbitrage problems, but care needs to be taken to properly measure the costs of ISPbound traffic. However, we show cost-based termination charges are only efficient in the unlikely case the regulated retail price of local calls is set at the cost of originating and terminating an ISP-bound call.

In cases where the retail prices of local calls are significant (such as in the UK and other European countries), bill and keep or cost based termination implies the price of Internet access will remain significant. One way to ensure a lower price for Internet dial-up is to force the incumbent to provide wholesale access in which ISPs or entrant carriers lease origination services from the incumbent local network and then provide their own dial-up service. Provided the per-minute component of any such leases is kept small (or zero), the per-minute price of Internet access will be similarly low. Alternatively, our proposed efficient termination charge also leads to low usage prices for ISP dial-up, even when the incumbent's retail price for such calls is high, since it is chosen to deliver total Internet dial-up prices based on underlying cost.

\section{Acknowledgements}

I would like to thank Henry Ergas, Alexis Harden and Emma Lanigan for insightful comments. I am also grateful to the Network Economics Consulting Group (NECG) for providing financial support for earlier related work. However, the views expressed here are strictly my own, as is the responsibility for any errors. 


\section{References}

Armstrong, M., 1998. Network interconnection. The Economic Journal 108, 545-564.

Atkinson, J., Barnekov, C., 2000. A Competitively Neutral Approach to Network Interconnection, OPP Working Paper No. 34. FCC.

Boston, W., 2000. Regulator investigates Deutsche Telekom for anticompetitive pricing suspicions, The Wall Street Journal, September 18th.

Carter, M., Wright, J., 2003. Asymmetric network interconnection. Review of Industrial Organization 22, $27-46$.

DeGraba, P., 2000. Bill and Keep at the Central Office as the Efficient Interconnection Regime, OPP Working Paper No. 33. FCC.

FCC, 2001a. FCC Initiates a Broad-Ranging Proceeding to Explore Ways of Reforming Intercarrier Compensation Rules, April 19. Action 01-132.

FCC, 2001b. Federal Communications Commission Resolves Carrier Compensaton Rules for Internet Traffic, April 19. Order 01-131.

Laffont, J.-J., Rey, P., Tirole, J., 1998. Network competition: I. Overview and nondiscriminatory pricing. RAND Journal of Economics 29, 1-37.

Randolph, W.S., 2000. Letter from Verizon Communications to Magalie R. Salas, Secretary, FCC, FCC Docket No. 99-68, November 1.

Wright, J., 2002. Access pricing under competition: An application to cellular networks. Journal of Industrial Economics L, 289-315. 